\title{
The contiguous domains of Arctic Ocean advection: trails of life and death
}

\author{
Paul Wassmann \\ Institute of Arctic and Marine Biology \\ Faculty of Biosciences, fisheries and economy \\ University of Tromsø - Norway's Arctic University \\ N-9037 Tromsø, Norway
}

We describe the circulation and transport of the Arctic Ocean and how advection from the Pacific and Atlantic Oceans support contiguous and macroecological domains. In particular we focus upon the distribution and advection of mesozooplankton and addresses a set of questions. How is the interaction between local versus advected "production" in the Arctic Ocean? Does advection make the Arctic Ocean a potentially net-heterotrophic region? What is the fate of the advected mesozooplankton biomass: trails of death or trails of life that feed hungry planktonic carnivores or the benthos? How will advection develop during climate warming? It is concluded that the mesozooplankton death march into the Arctic Ocean is part of the persistent invasion/ withdrawal battle of subarctic versus arctic species where death is a "calculated" risk for potential progeny. 